\title{
Review of: "Discovery of S-217622, a Non-Covalent Oral SARS-CoV-2 3CL Protease Inhibitor Clinical Candidate for Treating COVID-19"
}

\author{
Hiroyuki Konno ${ }^{1}$ \\ 1 Yamagata University
}

Potential competing interests: The author(s) declared that no potential competing interests exist.

This paper is described discovery of S-217622 for treating COVID-19. S-217622 is a non-covalent oral SARS-CoV2 3CL protease inhibitor for the first time and moreover become clinical candidate. S-217622 was discovered by the combination of virtual and biological screenings. S-217622 showed valuable PK profiles and became a drug candidate for the treating COVID-19. This paper is hot paper and gives us the exciting contents. Therefore, the reviewer agrees the release of bioRxiv. The reviewer has two comments.

1. P12-13, Figure 6 shows the good inhibitory activities of S-217622 against five corona-virus. Are their main proteases for the processing similar in the point of active site and substrate sequences? S-217622 has great potential for corona virus and strict selectivity against human host proteases.

2. Did the authors check the metabolites by clearance? It is important to show the metabolites. 